INPLASY

PROTOCOL

To cite: Xu et al. Acupuncture For Major Depressive Disorder: A systematic review and metaanalysis with trials sequential analysis. Inplasy protocol 2021120029. doi:

10.37766/inplasy2021.12.0029

Received: 04 December 2021

Published: 04 December 2021

Corresponding author:

Guixing Xu

1032159472@qq.com

Author Affiliation:

Chengdu University of

Traditional Chinese Medicine

Support: National Natural

Science Found.

Review Stage at time of this submission: Preliminary

searches.

Conflicts of interest:

None declared.

\section{Acupuncture For Major Depressive Disorder: A systematic review and meta-analysis with trials sequential analysis}

Xu, GX1; Xiao, QW2; Huang, BQ3; Lei, HZ4; Yin, ZH5; Huang, LY6; Zhou, Z7; Tian, H8; Huang, FY9; Liu, YL10; Sun, MS11; Zhao, L12; Liang, FR ${ }^{13}$; Li, $\mathrm{X}^{14}$.

Review question / Objective: Whether acupuncture is more benefit for MDD than antidepressant alone?

Condition being studied: Acupuncture therapy have been practiced in China for emotions disease. The WHO had analyzed the scope and efficacy of acupuncture therapy, and recommend acupuncture for depression with the level 1 evidence grade6. Previous evidence-based studies have indicated that acupuncture is associated with clinically relevant effect sizes in reducing the severity of depression when compared to sham-acupuncture. However, it is not clear whether acupuncture is more benefit for MDD than antidepressant alone. The randomized controlled trials, and meta-analysis cannot clear answer the question, because the small sample size with low statistic power, methodology defect, potential bias, etc. in RCTs, and include low quality RCTs, no strict methodology, and nonstandard report in metaanalysis.

INPLASY registration number: This protocol was registered with the International Platform of Registered Systematic Review and Meta-Analysis Protocols (INPLASY) on 04 December 2021 and was last updated on 04 December 2021 (registration number INPLASY2021120029).

\section{INTRODUCTION}

Review question / Objective: Whether acupuncture is more benefit for MDD than antidepressant alone?
Condition being studied: Acupuncture therapy have been practiced in China for emotions disease. The WHO had analyzed the scope and efficacy of acupuncture therapy, and recommend acupuncture for depression with the level 1 evidence grade6. Previous evidence-based studies 
have indicated that acupuncture is associated with clinically relevant effect sizes in reducing the severity of depression when compared to sham-acupuncture. However, it is not clear whether acupuncture is more benefit for MDD than antidepressant alone. The randomized controlled trials, and meta-analysis cannot clear answer the question, because the small sample size with low statistic power, methodology defect, potential bias, etc. in RCTs, and include low quality RCTs, no strict methodology, and nonstandard report in meta-analysis.

\section{METHODS}

Participant or population: Patients (aged $\geq$ 18 years) were diagnosed with MDD according to the third edition of Chinese Classification of Mental Disorders (CCMD-2/3), the International Statistical Classification of Diseases and Related Health Problems (ICD-10/11), or the Diagnostic and Statistical Manual of Mental Disorders (DSM-II/III/III-R/IV/IV-TR/V).

Intervention: Acupuncture.

Comparator: Antidepressant.

Study designs to be included: We searched EMBASE, The Cochrane Library, PubMed, The Cochrane Central Register of Controlled Trials, Web of Science, The China National Knowledge Infrastructure, The Wanfang database, The VIP Database for Chinese Technical Periodicals, The China Doctoral Dissertations Full-text Database for all randomized controlled trials (RCTs) published from inception to 10th September 2021.

Eligibility criteria: High quality randomized controlled trials (RCTs, Jadad score $\geq 4$ ) of eligible intervention(s) and outcome(s) for MDD published in Chinese and English languages were included. We excluded non-RCTs studies, non-efficacy evaluation studies, preclinical studies, studies comparing the efficacy of different acupoints in acupuncture therapy, low quality RCTs, trials of patients without clear diagnostic criteria; the conference proceedings or abstracts.All subjects (18 years and above) who meet one of the following diagnostic criteria for MDD irrespective of gender, race, or nationality: The third edition of Chinese Classification of Mental Disorders; International Statistical Classification of Diseases and Related Health Problems, ICD-1025; The Diagnostic and Statistical Manual of Mental Disorders, and DSM-II/III/III-R/IV/IV-TR/V. In this study, we included acupuncture as experiment group. There were no distinctions on manipulation methods, acupoint selection, needle retaining time, course of treatment and follow-up period. Comparators only include antidepressant medications. The primary outcome was treatment the grading of Hamilton Depression Rating Scale for Depression (HAMD17/24), reporting by the changes of four factors form baseline to after intervention scores in HAMD17/24(anxious/ somatization, grade of weight, sleeping and retardation factors). HAMD-17, to assess patients' activity that containing the items of depressed mood, guilt, work and interests, psychomotor retardation, psychic anxiety, and somatic general. The secondary outcome was adverse effect after intervention.

Information sources: The data of after intervention were extracted from the RCTs. The standard electronic data-extraction table were made through a general meeting, which including basic information of studies (author information, title, publication, etc.); characteristic of trial (study design, sample size, grouping method, etc.); the participants (age, gender, diagnosis, duration, etc.); method of intervention/control (number of treatment, frequency, duration of a session, etc.); outcome measurements (primary outcome, secondary outcome, etc.);results (mean, standard deviation (SD), adverse event, etc.). In addition, the extracted data were preserved in an Excel2016 form, and a third researcher (XQW) will check the input data to ensure the consistency and validity. All data were extracted by two authors (LHZ and $\mathrm{YZH}$ ) independently. 
Main outcome(s): Hamilton Depression Rating Scale for Depression.

\section{Additional outcome(s): Adverse effect.}

Quality assessment / Risk of bias analysis: The methodological quality of eligible trials were measured by the Risk of Bias Tool (ROB) in Cochrane Handbook (5.1.0) by two independent researchers (FYN and XQW). Each criterion was graded with three levels: low risk of bias, high risk of bias and unclear risk of bias. Lacking relative information for the assessment will urge us to contact the corresponding authors of the included trials for the required information. The methodology quality was assessed by two independent investigators (XQW and HBQ) with the Jadad scale. Any dissent occurs in the procedures were judged by a third investigator (YZH) after the cross-checking of study assessment.

Strategy of data synthesis: The paired meta-analysis, and cumulative metaanalysis were conducted by STATA 15.0 with random effects model. The standard mean differences (SMD) with $95 \%$ confidence interval $(\mathrm{Cl})$ were calculated for HAMD score, changes of HAMD. The TSA 0.9.5.10 $\beta$ software (Copenhagen Trial Unit, Copenhagen, Denmark) was used to perform the TSA for required information size (RIS) estimate with 0.05 for type I error $(a)$ and 0.2 for type II errors ( $\beta$ ). The random effects model of Biggerstaff, and Tweedie (BT), comprehensively consider the uncertainty of variance estimation between model tests of random effects, and clinical trials with large sample sizes were given more weight, was used for TSA. The invalid boundary was formed byßconsumption function of $O$ 'Brien-Fleming, the sequential monitoring boundaries formed by a consumption function of O'Brien-Fleming, the Z-curve was formed by cumulative Zvalue31, and RIS calculated by sample size estimation. The quality of evidence was assessed with the Grading of Recommendations Assessment, Development, and Evaluations (GRADE) system. The quality of evidence was rated as 4 levels: 'high', 'moderate', 'low' or 'very low'. At last, a summary of findings was presented.

Subgroup analysis: None.

Sensitivity analysis: We used the 12 to evaluated the heterogeneity, and the sensitivity analysis, and meta-regression was conducted to explore the source of heterogeneity when the $12>50 \%$. The funnel plot was used for digital-based modelling of the results. And the Egger's test was used to explore potential publication biases.

Country(ies) involved: China.

Keywords: Acupuncture, depression, paired meta-analysis, cumulative metaanalysis, trial sequential analysis.

Contributions of each author:

Author 1 - Guixing Xu.

Author 2 - Qiwei Xiao.

Author 3 - Biqing Huang.

Author 4 - Hanzhou Lei.

Author 5 - Zihan Yin.

Author 6 - Liuyang Huang.

Author 7 - Zhuo Zhou.

Author 8 - Hao Tian.

Author 9 - Fengyuan Huang.

Author 10 -Yilin Liu.

Author 11 - Mingsheng Sun.

Author 12 - Ling Zhao.

Author 13 - Fanrong Liang.

Author 14 - Xiang Li. 